\title{
Avaliação da Descentralização do Programa de Controle da Tuberculose do Nível Secundário para o Nível Primário do Sistema de Saúde de Dourados-MS
}

\section{Evaluation of the Decentralization of the Tuberculosis Control Program from Secondary to Primary Level in the Health System of Dourados-MS}

\author{
Arino Sales do Amaral \\ Enfermeiro. Mestre em Saúde Coletiva. Docente do Curso de Enfer- \\ magem da Universidade Estadual de Mato Grosso do Sul. \\ Endereço: Rua Camburiu, 15, BNH 3 Plano, CEP 79840-440, Dou- \\ rados, MS, Brasil. \\ E-mail: profarinoळuems.br

\section{Edson Mamoru Tamaki} \\ Doutor em Economia da Saúde. Docente da Universidade Federal \\ de Mato Grosso do Sul. Centro de Ciências Biológicas e da Saúde, \\ Departamento de Saúde Coletiva. \\ Endereço: Cidade Universitária, s/n, Caixa Postal 549, CEP 79070- \\ 900, Campo Grande, MS, Brasil. \\ E-mail: tamaki.msiळterra.com.br

\section{Cibele de Moura Sales} \\ Enfermeira. Doutora em Ciências da Saúde. Docente do Curso de \\ Enfermagem da Universidade Estadual de Mato Grosso do Sul. \\ Endereço: Rua Araguaia, 38, Jardim Água Boa, CEP 798॥-130, \\ Dourados, MS, Brasil. \\ E-mail: cm.salesळterra.com.br

\section{Rogério Dias Renovato} \\ Farmacêutico. Doutor em Educação. Docente do Curso de Enferma- \\ gem da Universidade Estadual de Mato Grosso do Sul. \\ Endereço: Rua Hilda Bergo Duarte, 296, CEP 79806-020, Dourados, \\ MS, Brasil. \\ E-mail: rrenovato®uol.com.br
}

\section{Resumo}

O objetivo deste estudo foi avaliar a descentralização das ações do controle da tuberculose do nível secundário para o nível primário do sistema de saúde de Dourados-MS, realizado através da implantação do Programa Nacional de Controle da Tuberculose (PNCT), no período de 2003 a 2006. A pesquisa constituiu-se em avaliação normativa com a utilização de dados secundários para obter o conhecimento do contexto e dos resultados das ações desenvolvidas. Foram utilizadas como categorias de análise: estrutura, processo e resultados. Ao analisar o processo de descentralização foi possível observar mudanças na atenção proporcionada aos usuários, não somente pela melhoria no acesso, mas, principalmente, pela postura dos profissionais de saúde evidenciada através de aumento significativo na busca dos sintomáticos respiratórios, na coleta de escarro para a baciloscopia e na detecção dos bacilíferos entre o total dos casos pulmonares encontrados. Todavia, os avanços verificados não trouxeram os resultados esperados para o município, indicando a existência de fragilidades ou lacunas de conhecimento que comprometeram os esforços dispensados no desenvolvimento de ações do programa.

Palavras-chave: Descentralização; Tuberculose; Atenção básica. 


\section{Abstract}

The objective of this study was to evaluate the decentralization of the actions for tuberculosis control from the secondary to the primary level in the health system of the city of Dourados (CentralWest region of Brazil), accomplished through the implementation of the Programa Nacional de Controle da Tuberculose (PNCT - Tuberculosis Control National Program), from 2003 to 2006. The research consisted of a normative evaluation with the use of secondary data in order to learn about the context and results of the developed actions. Structure, process and results were used as analysis categories. When analyzing the decentralization process, it was possible to observe changes in the care provided for users, concerning not only the improved access, but mainly the attitude of the health professionals, evidenced by a significant increase in the search for respiratory symptomatic individuals, in sputum collection for bacilloscopy and in the detection of bacillary patients among the total lung cases that were found. However, these advancements did not bring the expected results to the city, which indicates the existence of fragilities or knowledge gaps that compromised the efforts made in the development of the program's actions.

Keywords: Decentralization; Tuberculosis; Primary Health Care.

\section{Introdução}

O Programa Nacional de Controle da Tuberculose (PNCT), lançado pelo Ministério da Saúde em fevereiro de 2004, apresenta a tuberculose como uma doença de profundas raízes sociais que, além de estigmatizada como doença da pobreza, encontra-se agravada pela associação com a Síndrome da Imunodeficência Humana (AIDS) e o aparecimento de focos de tuberculose multidrogarresistente, tornando-se sério problema de saúde pública (Brasil, 2004). Uma pessoa doente infecta de dez a quinze pessoas anualmente, mas o infectado não necessariamente desenvolve a doença considerando que seu sistema imunológico competente destrói as bactérias ou as isola no local da infecção. Quando a imunidade está comprometida há grandes chances de adoecer pela reativação do bacilo inativo (Veronesi e Focacia, 2004).

Segundo a Organização Mundial de Saúde (OMS), estima-se que no Brasil - o $15^{\circ}$ entre os 22 países responsáveis por $80 \%$ dos casos de tuberculose no mundo - a prevalência seja de 50 milhões de infectados com cerca de 111 .ooo casos novos e 6.0oo óbitos anualmente (World Health Organization, 2005).

Apesar de a tuberculose (TB) ser uma doença que pode ser curada em seis meses, ela continua sendo um problema de saúde pública, pois seu controle não tem apresentado a evolução esperada pelas autoridades de saúde. Os índices da doença que diminuíram gradativamente na década de 1980 voltaram a crescer a partir dos anos 1990, associados também ao risco de aparecimento de bacilos resistentes. Esse cenário exigiu dos governos ações firmes e articuladas para controle, com adoção de estratégia de tratamento como forma de aumentar a detecção dos casos, assegurar a cura de todos os doentes, reduzir o abandono de tratamento e evitar o aumento da chamada resistência medicamentosa (Cheade, 2007)

Apesar de o Brasil ser o primeiro país do mundo a implantar o tratamento de curta duração de seis meses (DOTS - Direct Observed Therapy, Short Course), aqui conhecido como Tratamento Supervisionado, a tuberculose tem se expandido, em parte, como consequência da associação com o Vírus da Imunodeficiência Humana (HIV) (Brasil, 2001). 
A estratégia DOTS, proposta pela Organização Mundial de Saúde (OMS) aos órgãos responsáveis pela saúde de diversos países, aumenta a adesão dos pacientes, facilita a descoberta de maior número de fontes de infecção (paciente pulmonar bacilífero), aumenta a cura e reduz o risco de transmissão da doença na comunidade. A estratégia consiste na observação direta da tomada de medicamento para a tuberculose pelo menos uma vez por semana durante o primeiro mês de tratamento, na aquisição e distribuição regular de medicamentos e na criação de um eficiente sistema de informação (Brasil, 2002a).

No Brasil a taxa média de êxito do tratamento com a estratégia DOTS, em 2002, foi de $82 \%$, superior à média das regiões da África (73\%) e Europa (76\%), cujos resultados podem ser atribuídos em parte à coinfecção por HIV e à farmacorresistência, respectivamente, e também ao fracasso da estratégia DOTS na vigilância dos resultados dos tratamentos de todos os pacientes nessas regiões (Brasil, 2004).

Em 2004, a coordenação nacional do PNCT priorizou os 290 municípios brasileiros com maior carga da doença para implementar o controle da tuberculose. No Mato Grosso do Sul, foram incluídos cinco municípios: Amambaí, Campo Grande, Corumbá, Dourados e Três Lagoas (Brasil, 2004).

O município de Dourados, situado na região sul de Mato Grosso do Sul foi priorizado pelo Ministério da Saúde para o controle da tuberculose por apresentar um coeficiente de incidência da tuberculose no período de 2000-2002 superior a 47/100.000 habitantes, possuir uma população maior que 100 mil habitantes, possuir uma população indígena fazendo parte do município, e se encontrar entre os municípios brasileiros que precisavam consolidar as ações do PNCT na Atenção Básica (Brasil, 2004).

Quando foi iniciada em 2004 a implementação do PNCT em Dourados-MS, as atividades de controle da tuberculose para todo o município eram realizadas centralizadamente através de duas unidades do nível secundário do sistema de saúde. As 28 Unidades Básicas de Saúde (UBS) existentes no município não possuíam atribuições específicas para esse fim.

Nesse mesmo ano, foi decidida a descentralização das atividades de controle da tuberculose para o nível primário, implantando o PNCT nas UBS do município. A expectativa dos responsáveis pela saúde era que com a estratégia haveria uma melhoria no controle da doença e, consequentemente, nos indicadores de morbidade e mortalidade do município.

No entanto, conforme foi observado anteriormente, apesar de existir um tratamento eficaz, a tuberculose continua sendo um problema de saúde pública. Isso nos permite constatar que não há garantia de que a implantação do PNCT atinja os objetivos previstos. Por isso, a avaliação de programas de saúde constitui um instrumento capaz de trazer a resposta para essa questão.

A avaliação de programas de saúde constitui um processo estruturado e lógico de obtenção de conhecimento da realidade e de confrontação com valores de referência (normas, modelos teóricos, parâmetros entre outros) que permitem emitir um julgamento de valor sobre um objeto determinado. Em se tratando de um programa de saúde, a avaliação tem como foco a análise dos processos complexos de organização de práticas voltadas para objetivos específicos, como a implantação de formas de atenção para populações específicas (Novaes, 200o; Matida e Camacho, 2004).

Visando buscar resposta para esse questionamento, o objetivo deste estudo foi avaliar a descentralização das ações do controle da tuberculose do nível secundário para o nível primário do sistema de saúde de Dourados-MS, realizado através da implantação do PNCT.

\section{Método}

Trata-se de avaliação normativa, que se consistiu na aplicação de critérios e normas (Contandriopoulos, 2006) e na utilização de dados secundários para obter o conhecimento do contexto e dos resultados das ações desenvolvidas. Foram utilizadas as seguintes categorias de análise: estrutura, processo e resultados, as mesmas preconizadas pelo Ministério da Saúde para efetuar o acompanhamento do PNCT (Brasil, 2004). Estas categorias, definidas por Donabedian (1988) para a avaliação em saúde, tem uma correspondência direta a momentos distintos da implementação de programas de saúde (Mallet, 2005).

A categoria estrutura corresponde às pré-condi- 
ções necessárias para a execução de um programa, como: recursos humanos, materiais, financeiros e organizacionais. 0 processo corresponde à fase de execução propriamente dita, e o resultado corresponde à fase de obtenção dos efeitos das ações e atividades realizadas sobre a saúde dos indivíduos e da população (Donabedian, 1988; Mallet, 2005; Santos-Filho, 2007).

A validade dessa tríade na avaliação de programas de saúde depende da existência de relações causais entre elas, ou seja, uma estrutura adequada possibilita bom processo que conduz a resultados satisfatórios (Donabedian, 1988). Entretanto, em face da multiplicidade de fatores que condicionam a saúde, essas relações não são totalmente conhecidas o que faz com que a concepção de programas seja baseada em pressupostos que não garantem obrigatoriamente a obtenção dos resultados pretendidos.

Nessas condições, a avaliação de programas de saúde constitui uma atividade essencial para verificar se os objetivos foram atingidos e em que medida os resultados pretendidos foram obtidos.

Considerando que a descentralização do PNCT para as unidades básicas de saúde foi realizada no período de 2004 a 2005 , foi escolhido o período de 2003 a 2006 para ser objeto de estudo. 0 objetivo foi obter informações da situação do controle da tuberculose um ano antes do início e um ano após o término do processo de descentralização em Dourados.

A população beneficiada pela descentralização do PNCT foi a da área de abrangência da rede básica do município, denominada neste estudo de população residente. Por esse motivo a população indígena aldeada e a população carcerária existente no município foram excluídas do estudo, pois possuem sistema próprio de atenção à saúde, não sendo, portanto, beneficiadas pelo processo de descentralização do controle de tuberculose.

Para a obtenção de dados sobre a tuberculose no município foi necessário realizar um exaustivo levantamento nos registros dos serviços de saúde devido à falta de dados organizados ou sistematizados. Além dos dados do Sistema de Informação de Agravos de Notificação (Sinan) e do Sistema de Informação da Atenção Básica (SIAB), foram utilizados como fonte de dados secundários:
1. Plano de Ação do Programa Nacional de Controle da Tuberculose do Município de Dourados/MS - 2005-2007.

2. Livro de Registro e Controle de Tratamento dos Casos de Tuberculose da Coordenação do Programa de Tuberculose.

3. Livro de Registro de Sintomáticos Respiratórios com o número de atendimentos do PNCT registrados no Sistema de Informação da Atenção Básica (SIAB).

4. Relação do número de baciloscopias realizadas para diagnóstico, de 2003 a 2006.

5. Relatório de conferência do Sinan.

6. Relatório do resultado de baciloscopias dos sintomáticos respiratórios realizadas no Centro de Saúde de Dourados e Hospital Universitário.

7. Relatório de cobertura Vacinal em menores de um ano do Programa Nacional de Imunização (PNI).

8. Estudo do resultado de tratamento das coortes de encerramento de casos novos de tuberculose em Dourados/MS de 1996-2006 da Secretaria de Estado de Saúde.

Objetivando determinar os efeitos da descentralização do controle da tuberculose para o nível primário do sistema de saúde foi feita a comparação da situação verificada na coorte de 2006 com a situação existente na coorte de 2003. De acordo com o Ministério da Saúde (Brasil, 2006), para a tuberculose a coorte é designada por um grupo de pacientes (casos novos), com início de tratamento (de duração de seis meses) em determinado período.

\section{Resultados e Discussão}

No ano de 2002, o município de Dourados apresentava a incidência de tuberculose de 50,8/100 mil habitantes. A redução de casos ocorreu nos anos subsequentes, porém entre 2005 e 2006 houve um ligeiro aumento, passando para 37,6 e 39,7 casos por 100 mil habitantes, respectivamente (Tabela 1). Esses coeficientes foram calculados considerando a população total do município.

O cálculo da incidência de tuberculose na população residente do município de Dourados, separada da incidência de populações não beneficiadas pela descentralização do PNCT (população indígena alde- 
ada e população carcerária), revela que a incidência de tuberculose (22,5/100.0oo habitantes em 2006) (Tabela 1) é muito inferior ao preconizado pelo Ministério da Saúde para considerar Dourados como município prioritário no PNCT (47/100.ooo habitantes) e abaixo do coeficiente de incidência nacional de 45 casos por 100 mil habitantes (Dominguez,
2006), ou seja, se as populações vulneráveis que possuem sistemas particulares de atenção à saúde fossem excluídas do cálculo da incidência geral da tuberculose de um município, poderia haver uma modificação na relação dos municípios enquadrados como prioritários para o PNCT, como seria o caso do município de Dourados.

\section{Tabela I - Incidência de tuberculose na população residente de Dourados-MS, 2002 a 2006}

\begin{tabular}{lccccc} 
ANO & 2002 & 2003 & 2004 & 2005 & 2006 \\
População total & 171.042 & 173.874 & 176.693 & 183.097 & 186.357 \\
Incidência população total & 50,8 & 41,4 & 35,6 & 37,6 & 39,7 \\
População residente & 159.847 & 162.139 & 164.708 & 170.723 & 173.353 \\
Incidência população residente & 35,6 & 22,8 & 20 & 22,8 & \\
\hline
\end{tabular}

Fonte: Brasil, 2006.

Em Dourados, observou-se uma redução de incidência de tuberculose na população residente a partir de 2002 estabilizando-se em um patamar de 22,8 e 22,5 casos por 100 mil habitantes nos anos de 2005 e 2006. Esses coeficientes foram, no entanto, menores que o coeficiente de incidência de Mato Grosso do Sul nos 12 últimos anos, que foi de $43 \mathrm{ca}$ sos por 100 mil habitantes em média (Mato Grosso do Sul, 2006).
A análise dos indicadores da estrutura reflete a evolução da descentralização do PNCT no período de 2003 a 2006. Nesse último ano, 92,8\% das unidades básicas de saúde já apresentavam o programa da tuberculose implantado e todos com tratamento supervisionado oferecido (Tabela 2). Porém, ainda abaixo da meta preconizada pelo PNCT, que estabelecia para esse ano a implantação do programa em 100\% das unidades básicas de saúde do município (Mato Grosso do Sul, 2006).

Tabela 2 - Indicadores de estrutura do Programa Nacional de Controle da Tuberculose em Dourados-MS, 2003-2006

\begin{tabular}{|c|c|c|c|c|}
\hline AÇÃO/ ANO & 2003 & 2004 & 2005 & 2006 \\
\hline Percentual de unidades básicas de saúde com PNCT implantado & - & 37 & 70,2 & 92,8 \\
\hline $\begin{array}{l}\text { Percentual de unidades básicas de saúde com tratamento } \\
\text { supervisionado dentre as unidades com PNCT implantado }\end{array}$ & - & 100 & 100 & 100 \\
\hline $\begin{array}{l}\text { Laboratórios da rede do Sistema Único de Saúde (SUS) que } \\
\text { executam baciloscopia }\end{array}$ & 4 & 4 & 4 & 4 \\
\hline
\end{tabular}

Fonte: Dourados, 2007. 
A principal mudança observada nos indicadores de estrutura foi o crescimento da cobertura das unidades básicas de saúde com o programa de tuberculose implantado, incluindo o tratamento supervisionado. A evolução do percentual de unidades básicas de saúde com tratamento supervisionado foi de 37\% em 2004, 70,2\% em 2005 e 92,8\% em 2006. Os laboratórios da rede de saúde pública permaneceram sem alteração nesse período, pois a sua capacidade instalada era suficiente para atender a demanda existente.

Os indicadores de processo mostraram que houve melhoria das atividades de controle da tuberculose no período estudado. A taxa de cobertura vacinal com BCG manteve-se em torno dos $100 \%$, conforme previsto pelo PNI. O número de sintomáticos respiratórios (SR) examinados aumentou significativamente na medida em que o programa de tuberculose estava sendo descentralizado. Comparando 2003 com o ano de 2006, observou-se um aumento de $81,6 \%$ (Tabela 3).

Em 2006, a proporção de casos de tuberculose pulmonar com baciloscopia realizada foi de $71,6 \%$. Apesar de o município ter realizado esse exame em todos os casos de tuberculose pulmonar, o PNCT só considera os exames com resultados positivos. Em relação ao ano de 2003, o ano de 2006 apresentou um aumento nessa proporção de $86,5 \%$, no entanto, a meta de $90 \%$ estabelecida para esse ano não foi alcançada (Brasil, 2004).

Quanto à proporção de bacilíferos entre o total de casos pulmonares encontrados, observou-se avanço de 71,2 \% no período de 2003 a 2006, pois essa proporção passou de $48,3 \%$ para $82,7 \%$, superior à meta do PNCT que era de 70\% dos bacilíferos para 2006 (Brasil, 2004).

\section{Tabela 3 - Indicadores de processo do Programa Nacional de Controle da Tuberculose em Dourados-MS, 2003 a 2006}

\begin{tabular}{|c|c|c|c|c|}
\hline AÇÃO/ ANO & 2003 & 2004 & 2005 & 2006 \\
\hline Taxa de cobertura vacinal (\%) com BCG em menores de um ano & 105,9 & 107,8 & 102,9 & 91 \\
\hline Número de sintomáticos respiratórios examinados & 429 & 480 & 625 & 779 \\
\hline Proporção de casos (\%) de TB pulmonar com baciloscopia realizada & 38,4 & 50 & 80,3 & 71,6 \\
\hline Proporção de bacilíferos (\%) entre o total de casos de TB pulmonar & 48,3 & 46,1 & 74,1 & 82,7 \\
\hline Número de UBS com profissionais capacitados em ações/atividades do PNCT & 1 & 14 & 26 & 26 \\
\hline $\begin{array}{l}\text { Percentual de casos detectados de tuberculose dentre o total de casos } \\
\text { estimados }\end{array}$ & 47,6 & 46,6 & 64,7 & 61,2 \\
\hline
\end{tabular}

Fonte: Dourados, 2007; Mato Grosso do Sul, 2007.

O número de unidades com profissionais capacitados em ações do PNCT aumentou no período de 2003 a 2006, indicando a expansão do programa. Em 2003, havia apenas uma unidade com profissionais capacitados e, em 2006, esse número já atingia 26 unidades básicas de saúde.

0 percentual de casos detectados de tuberculose em relação ao total de casos estimados foi de $47,6 \%$ em 2003 e 67,2\% em 2006, no entanto ainda distante dos 79,1\% estabelecidos como meta do PNCT a ser atingido em 2006 (Brasil, 2004).
Na procura de casos, além da busca dos sintomáticos respiratórios, foi importante o controle de contatos: pessoas, parentes ou não, que coabitavam com um doente de tuberculose. Os comunicantes de pacientes bacilíferos e crianças eram prioritários para a ação de busca de casos (Brasil, 2002a). Em Dourados, o controle dos contatos era realizado pelas equipes das unidades, contatos de bacilíferos e contatos de pacientes com diagnóstico não confirmado, assim que descoberto o caso. No relatório de conferência do Sistema de Informação de Notifica- 
ção de Agravos (Sinan) da Coordenação Municipal de Programa de Tuberculose (Dourados, 2006), foram realizados em 2003, quatro exames de contatos, em 2004, 228, em 2005, 93, e em 2006, 120.

No relatório de resultado de baciloscopias do laboratório do Centro de Saúde de Dourados, em 2006, constatou-se que foram encaminhados 343 exames de escarro para diagnóstico de tuberculose. O plano de ação do PNCT de Dourados (2007) mostrou que $70,2 \%$ das unidades fizeram coleta de escarro, um aumento relevante em relação ao ano de 2003, quando apenas uma unidade era responsável por essa coleta.

As coortes de encerramento apontaram baixo percentual de cura (Tabela 4), não atingindo em 2006 os $81 \%$ esperados pelo PNCT (Brasil, 2004a). Esse percentual foi o pior do período. Cabe ressaltar que essa meta também não foi atingida pelo estado de Mato Grosso do Sul, que registrou um percentual de cura não ultrapassando os $73 \%$ (Mato Grosso do Sul, 2006).

\section{Tabela 4 - Indicadores de resultados do Programa Nacional de Controle da Tuberculose em Dourados-MS, 2003 a 2006}

\begin{tabular}{l|cc|cc|cc|cc}
\multirow{2}{*}{ Coorte*/Ano } & \multicolumn{2}{|c|}{2003} & \multicolumn{2}{|c|}{2004} & \multicolumn{2}{c|}{2005} & \multicolumn{2}{c}{2006} \\
\cline { 2 - 9 } & N & $\%$ & N & $\%$ & N & $\%$ & N & $\%$ \\
Cura & 49 & 75,3 & 32 & 66,6 & 29 & 76,3 & 25 & 64,2 \\
Abandono & 05 & 7,7 & 9 & 18,8 & 4 & 10,5 & 4 & 10,2 \\
Óbito & 2 & 3,0 & 4 & 8,3 & 4 & 10,5 & 6 & 15,4 \\
Transferência & 9 & 14,0 & 3 & 6,3 & 1 & 2,7 & 4 & 10,2 \\
\hline Total & 65 & 100 & 48 & 100 & 38 & 100 & 39 & 100 \\
\hline
\end{tabular}

Fonte: Sistema de Informação de Agravos de Notificação (Sinan)

* Coorte de encerramento de casos novos com seis meses tratamento da tuberculose.

A análise dos indicadores de resultados do tratamento dos pacientes com tuberculose não refletiu a evolução positiva demonstrada pelos indicadores de estrutura e processo. Não houve melhoria do percentual de abandono com a descentralização do PNCT para as unidades, pois o percentual de abandono em 2006 (10,2\%) foi maior que o observado em 2003 (7,7\%), único ano em que o resultado do município superou a meta estabelecida pelo PNCT (12,0\%). Esperava-se que com o acompanhamento pela equipe de saúde da família, aumentasse a adesão ao tratamento e a queda no percentual de abandono, atingindo a meta de $7 \%$ esperado pelo PNCT em 2006. 0 percentual de abandono é o indicador que avalia a adesão e a qualidade do programa (Brasil, 2002b)

Percebeu-se piora nos percentuais de óbitos das coortes de encerramento do período estudado. Em 2006, o percentual de óbitos foi de $15,4 \%$, o mais alto do período, bem acima da meta de $5 \%$ estimada pelo Ministério da Saúde (Tabela 4). Esse indicador é importante no acompanhamento do tratamento e aponta a necessidade de identificar as causas que levam ao óbito, para que a equipe de saúde interfira no processo, diminuindo principalmente as causas de óbitos por tuberculose.

\section{Considerações Finais}

Ao analisar o processo de descentralização do PNCT para as unidades básicas de saúde de Dourados, verificaram-se avanços importantes. As ações de controle da tuberculose antes desenvolvidas em nível secundário, através de duas unidades de referência, foram transferidas para o nível primário, passando a ser desenvolvida em 92,8\% das unidades básicas de saúde do município.

No que se refere às questões de estrutura, o município capacitou profissionais em todas as unidades básicas de saúde, onde o PNCT foi implantado, assim como o tratamento supervisionado.

Quanto ao processo, houve melhorias significativas no volume de atividades com o aumento do 
número de SR examinados, de baciloscopias realizadas, detecção de novos casos, controle de contatos, aproximando-se das metas estabelecidas pelo PNCT, e até superando em alguns casos.

Foi possível observar mudanças na atenção proporcionada aos usuários, não somente pela melhoria no acesso ao PNCT, mas principalmente pela postura dos profissionais de saúde evidenciada através de aumento significativo na busca dos SR, na coleta de escarro para a baciloscopia e na detecção dos bacilíferos entre o total dos casos pulmonares encontrados.

As melhorias observadas na estrutura e no processo do programa não trouxeram as melhorias esperadas nos resultados. Apesar da leve melhoria no percentual de cura, houve uma piora no percentual de abandonos e aumento importante no percentual de óbitos.

Apesar do avanço do processo de descentralização, a utilização do tratamento supervisionado, procedimento comprovadamente eficaz no controle da tuberculose, não trouxe os resultados esperados para o município, indicando a existência de fragilidades ou lacunas de conhecimento, que comprometeram os esforços dispensados no desenvolvimento de ações do programa.

O PNCT em Dourados no período estudado avançou na descentralização contando com estrutura, insumos e profissionais capacitados para seu desenvolvimento, no entanto, o que se constatou foi a necessidade de novos estudos que tragam elementos que permitam compreender as causas dos limitados resultados obtidos e buscar soluções que permitam um efetivo controle da tuberculose no município.

\section{Referências}

BRASIL. Ministério da Saúde. Plano nacional de mobilização e intensificação das ações para a eliminação da hanseníase e controle da tuberculose. Brasília: Ministério da Saúde, 2001.

BRASIL. Ministério da Saúde. Manual técnico para o controle da tuberculose: cadernos de atenção básica. 6. ed. Brasília: Ministério da Saúde, 2002a. (Série A. Normas e Manuais Técnicos; n. 148).
BRASIL. Ministério da Saúde. FUNASA. Tuberculose: guia de vigilância epidemiológica. Brasília: Ministério da Saúde; FUNASA, 2002b.

BRASIL. Ministério da Saúde. Programa nacional de controle da tuberculose. Brasília: Ministério da Saúde, 2004.

BRASIL. Ministério da Saúde. Secretaria de Vigilância em Saúde. Roteiro para análise da base de dados de tuberculose do sistema de informação de agravos de notificação - SINAN e cálculo de indicadores básicos. Brasília: Ministério da Saúde, 2006.

CHEADE, M. F. M. Evolução clínica da tuberculose em pacientes infectados por HIV em Campo Grande-MS 2003-2005. 2007.122 f. Tese (Doutorado em Ciências da Saúde) - Programa de Pós-Graduação Multiinstitucional em Ciências da Saúde, Convênio Rede Centro-Oeste UNB/UFG/ UFMS, Campo Grande-MS, 2007.

CONTANDRIOPOULOS, A. P. Avaliando a institucionalização da avaliação. Ciência \& Saúde Coletiva, Rio de Janeiro, v. 11, n. 3, p. 705-711, jul.set. 2006.

DOMINGUEZ, B. C. Tuberculose: a cura está nas políticas públicas. Radis Comunicação em Saúde, Rio de Janeiro, n. 48, p. 14-15, ago. 2006.

DONABEDIAN, A. The quality of care. How can it be assessed? Journal of the American Medical Association, Chicago, v. 26o, n. 12, p. 1743-1748, sept. 1988.

DOURADOS (Município). Secretaria Municipal de Saúde. Série histórica de atendimento do programa de tuberculose. Dourados, MS: Sistema de Informação da Atenção Básica (SIAB), 2006.

DOURADOS (Município). Secretaria Municipal de Saúde. Plano de ação do programa de controle da tuberculose no município de Dourados-MS, 20052007. Dourados, MS: Secretaria Municipal da Saúde, 2007.

MALLET, A. L. R. Qualidade em saúde: tópicos para discussão. Revista da SOCERJ, Rio de Janeiro, v. 18 , n. 15 , p. $449-456$, set.-out. 2005. 
MATIDA, A. H.; CAMACHO, L. A. B. Pesquisa avaliativa e epidemiologia: movimentos e síntese no processo de avaliação de programas de saúde. Cadernos de Saúde Pública [online], Rio de Janeiro v. 20, n.1, p. 37-47, jan.-fev. 2004. Disponível em: <http://www.scielo.br/pdf/csp/v2on1/17.pdf >.

Acesso em: 12 maio 2010.

MATO GROSSO DO SUL. Secretaria de Estado de Saúde. Informe epidemiológico do programa de eliminação de hanseníase e controle da tuberculose. Campo Grande, Ano 1, n. 1, nov. 2006.

MATO GROSSO DO SUL (Estado). Secretaria de Estado de Saúde. Núcleo Regional de Saúde de Dourados. Relatório de cobertura vacinal em menores de um ano do Programa Nacional de Imunização de 2001 a 20o6. Dourados-MS: Secretaria de Estado de Saúde, 2007.
NOVAES, H. M. D. Avaliação de programas, serviços e tecnologias em saúde. Revista de Saúde Pública, São Paulo, v. 24, n. 5, p. 547-59, out. 2000.

SANTOS-FILHO, S. B. Perspectivas da avaliação na Política Nacional de Humanização em Saúde: aspectos conceituais e metodológicos. Ciência \& Saúde Coletiva, eRio de Janeiro, v. 12, n. 4, p. 9991010, jul.-ago. 2007.

VERONESI, R.; FOCACIA, R. Tratado de infectologia. 2. ed. São Paulo: Ateneu, 2004.

WORLD HEALTH ORGANIZATION. Tuberculosis: infections and transmission, 2005. Disponível em: <htpp://www.who.int/tb/en/>. Acesso em: 29 abr. 2005 . 